\section{政}

\title{
Deriving injury risk curves using survival analysis from biomechanical experiments
}

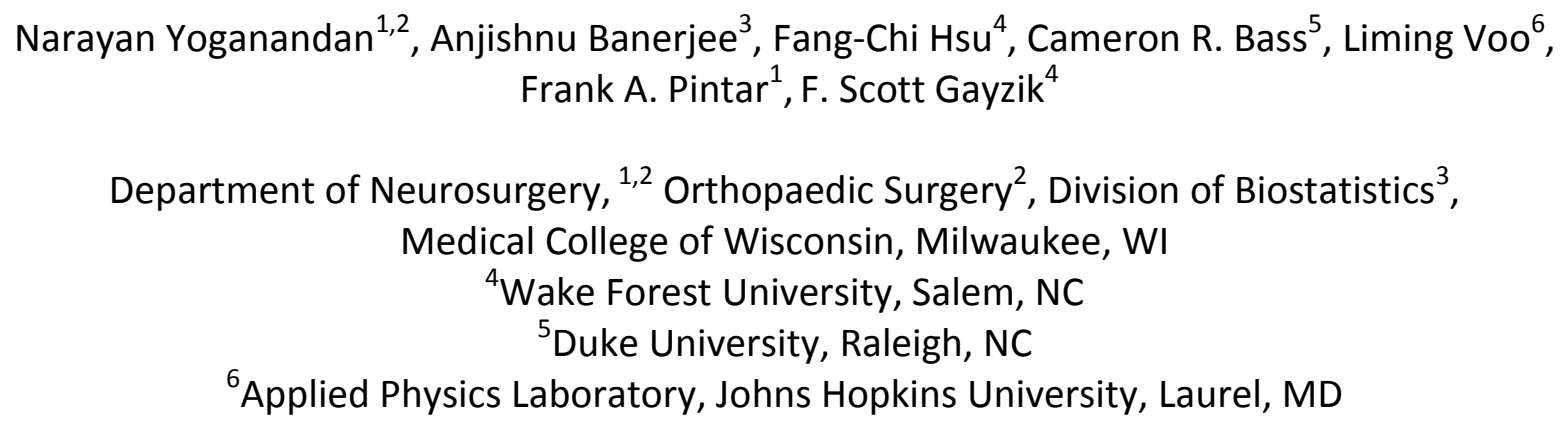

Running head: Injury risk curves from biomechanical experiments

Number of words Abstract: 250 (limit 250)

Number of words from Introduction to Discussion: 3475 (limit 3500)

\section{Correspondence:}

Narayan Yoganandan, PhD

Professor of Neurosurgery and Orthopaedic Surgery

Chair, Biomedical Engineering

Department of Neurosurgery

Medical College of Wisconsin

Milwaukee, WI 53226

Tel: 414-384-3453

Fax: 414-955-0115

yoga@mcw.edu 


\section{Abstract}

Injury risk curves from biomechanical experimental data analysis are used in automotive studies to improve crashworthiness and advance occupant safety. Metrics such as acceleration and deflection coupled with outcomes such as fractures and anatomical disruptions from impact tests are used in simple binary regression models. As an improvement, the International Standards Organization suggested a different approach. It was based on survival analysis. While probability curves for side-impact-induced thorax and abdominal injuries and frontal impact-induced foot-ankle-leg injuries are developed using this approach, deficiencies are apparent. The objective of this study is to present an improved, robust and generalizable methodology in an attempt to resolve these issues. It includes: (a) statistical identification of the most appropriate independent variable (metric) from a pool of candidate metrics, measured and or derived during experimentation and analysis processes, based on the highest area under the receiver operator curve, (b) quantitative determination of the most optimal probability distribution based on the lowest Akaike information criterion, (c) supplementing the qualitative/visual inspection method for comparing the selected distribution with a nonparametric distribution with objective measures, (d) identification of overly influential observations using different methods, and (e) estimation of confidence intervals using techniques more appropriate to the underlying survival statistical model. These clear and quantified details can be easily implemented with commercial/open source packages. They can be used in retrospective analysis and prospective design of experiments, and in applications to different loading scenarios such as underbody blast events. The feasibility of the methodology is demonstrated using post mortem human subject experiments and 24 metrics associated with thoracic/abdominal injuries in side-impacts. 
Paper to be submitted to Journal of Biomechanics

55 Key words: biomechanical experiments, confidence intervals, impact loading, probability 56 curves, survival analysis

57 
Paper to be submitted to Journal of Biomechanics

\section{Introduction}

Injury risk curves for military and automotive crashworthiness evaluations and for advancing occupant safety are developed using data from post mortem human subject (PMHS) experiments (Arbogast and Maltese, 2015; Petitjean et al., 2015; Rupp, 2015; Salzar et al., 2015; Wang et al., 2015; Yoganandan et al., 2014; Yoganandan et al., 2016; Yoganandan et al., 2013). Simple binary regression models have been used in the literature (Cavanaugh and Yoganandan, 2015; Kuppa et al., 2003; Philippens et al., 2009; Seipel et al., 2001). Survival analyses with different censoring assumptions have been used to develop injury models in biomechanics (Cutcliffe et al., 2012; Kent and Funk, 2004). The ISO/TC22/SC12/WG6 working group of the International Standards Organization (ISO) evaluated parametric and non-parametric methods used by researchers in the automotive field and suggested a unified and improved approach to obtain injury risk curves from retrospective PMHS tests. Survival analysis was recommended because of its ability to accommodate differing censoring status from experimental outcomes. This approach has the substantial advantage of incorporating the biomechanical knowledge beyond simple sensor data into the analysis.

Steps in the ISO approach are: (1) collecting data, (2) assigning censor status, (3) checking for multiple injury mechanisms, (4) separating samples by injury mechanism, (5) estimating distribution parameters, (6) identifying overly influential observations, (7) checking the distribution assumption, (8) choosing the distribution, (9) checking the validity of predictions against existing results, (10) calculating 95\% confidence intervals, (11) assessing the quality index, and (12) recommending one curve per body region (Petitjean et al., 2012). The 
suggested approach has been applied to derive risk curves for thoracic and abdominal injuries in nearside-impacts (Petitjean et al., 2012). In addition, survival analysis-based probability curves for foot-ankle-leg injuries resulting from the axial loading mechanism differed from simple logistic regression-based risk curves (Yoganandan et al., 2014). While the 12 steps in the ISO procedure appear to be detailed, they do not address all the nuances of the problem and deficiencies.

First, the ISO approach does not discuss the choice of biomechanical parameters for the model since it is common to gather more than one metric in any experiment. The Akaike information criterion (AIC) is the suggested in the ISO approach to choose a statistical distribution. The criterion is an ordinal score which can be used as a simple tool to rank probability distributions, for example, Weibull, log-normal and log logistic. The AIC scores can however, over-fit in a statistical model, and is known in the statistical literature to be asymptotically inconsistent (Findley and Ching-Zong, 2002). The ISO approach also recommends comparing the chosen distribution with a non-parametric fit through visual examination of the Quintile-Quintile (Q-Q) plot and an overlay of the fitted risk curve to a non-parametric risk curve. However, it does not suggest any quantitative measure for comparison.

Further, the ISO recommends the use of the dfbetas statistic to determine overly influential observations. The statistic is a measure of the influence of a data point for each model parameter. It corresponds to the difference between the parameter value when calculated with the data point present and the value when the data point is dropped from the sample. Several versions of this statistic corresponding to different methods of computation are 
available. The ISO approach does not suggest methods for identifying outlier(s). The calculation of confidence intervals for survival regression generally relies on asymptotic approximations, for which many established methods exist. The ISO approach does not specify the method to determine confidence intervals.

The objective of this study is to improve these procedures and provide a robust statistical framework to derive risk curves for injury biomechanics and other applications. This effort is attempted to resolve fundamental ambiguities in the ISO approach for deriving risk curves, and it demonstrates the feasibility using an example from published set of experiments. This procedure can be used in sub-component and whole body PMHS tests and for applications to different loading environments including military situations such as underbody blast events, automotive and others. All statistical analysis described can be implemented with commercial statistical packages such as SAS and public license software such as R-code.

\section{Methods}

The following steps describe the improved methodology. The flow chart is provided (Figure 1). The words risk and probability are used synonymously throughout the paper.

\section{Input data}

Input data includes PMHS information such as demographics, external loading scenarios, injury outcomes and biomechanical metrics. Demographic data such as age and sex, bone mineral density, body mass index, and certain specimen anthropometry (bone or spinal column length for sub-component and stature for whole body PMHS experiments) can serve as categorical or 
Paper to be submitted to Journal of Biomechanics

125

126

127

continuous co-variates. Loading scenarios such as single or repeated applications of the external load can be used to associate the injury or injury severity identified from the experiment with the biomechanical metric. Biomechanical metric(s) can be an individual parameter such as the peak force, deflection, or, combined parameters such as accelerations from different levels of the ribs and spine, associated with the injury mechanism/experiment under consideration. Metrics with no injury are considered as right censored. The metric is treated as left censored if the timing of injury is unknown. In contrast, it is considered as exact or uncensored if the experimental design, instrumentation and signal analysis allow the determination of the precise timing of injury. If repeated tests are included in the experimental design, interval censoring can be used with non-injury and injury outcomes: right censoring associated with the highest severity non-injury test and left censoring associated with the injury test. However, if the repeated testing protocol does not result in injury, the metric from the highest severity test is used as right censored.

1. Choose the most optimal biomechanical metric based on highest Area under the Receiver Operator Curve (AROC): Though multivariate survival analyses have been developed, survival regression often uses one variable as the primary independent variable (often the time variable in medical statistical analyses) (Guo and Rodriguez, 1992). In the presence of several biomechanical metrics, it is therefore important to decide which variable is most likely to produce the best statistical model - the variable best able to distinguish between injury outcomes. 
The proposed methodology involves the determination of the AROC for all biomechanical metrics. It represents a measure of the predictive ability of the metric in terms of specificity and sensitivity. The biomechanical metric producing the highest $A R O C$ is the most appropriate metric for further analysis.

2. Choose the most optimal candidate probability distribution based on lowest AIC: The next step is to choose the most appropriate probability distribution to be used with survival regression, which is typically done under a non-parametric (distribution free) or under a parametric setup. A parametric setup is often advantageous because of its flexibility and generally results in smoother risk models, i.e., accommodation of differing censor status and covariate combinations (Royston and Parmar, 2002). However, the parametric setup requires additional assumptions: most importantly that the unknown data generating distribution is close to the statistical distribution being used. The Weibull, log-normal and log-logistic are the commonly used distributions with survival regression. For each of these distributions, it is possible to assess fit and the distance from the non-parametric model (described later).

The proposed methodology calculates the AIC for each of the standard probability distributions used in the parametric survival regression models. The candidate probability distribution with the lowest AIC is identified as the most optimal distribution function among the candidates. 
3. Compare with Kolmogorov-Smirnov (KS) tests: The proposed methodology involves calculating the KS p-value for that distribution with the lowest AIC. The goodness-of-fit test (based on the distance of the fitted candidate from the non-parametric fit) with very low $p$-values, i.e., $p<0.05$, indicate a poorly fitting distribution. If the $p$-value exceed the set criterion $(p=0.05)$, the next step would be to identify the overly influential observations, described in 4 , below. If not, the distribution with the next higher AIC is selected (e.g., log-logistic), and the process is repeated. If all distributions do not meet the criterion, then, the metric associated with the next highest AROC is selected and the entire stated process is repeated, 1 , above.

4. Identify overly influential observations based on the dfbetas statistic: This involves deleting one observation/data point 'at a time' and determining the dfbetas statistic. The statistic corresponds to the difference between the coefficients of the model with the inclusion of all data points and the coefficient with one data point excluded from the ensemble. It can be computed on the basis of any moment or parameter of the chosen probability distribution.

The proposed improved methodology involves computing the statistic based on the location, shape and scale. The excluded data point is considered to be overly influential when the value of the statistic exceeds the magnitude defined as follows: $2 /$ square root of the sample size of the ensemble (Belsley et al., 1980). All overly influential sample points might represent erroneously recorded observations. Therefore, they all should 
be checked carefully for accuracy by the team that conducted the actual experiments and raw data analysis. In the absence errors, the experimental group and the subject matter expert should decide regarding the exclusion of any of these overly influential observations, while underscoring that exclusion(s) might lead to leptokurtic distributions and under-represented data variance.

5. Determine confidence intervals $(\mathrm{Cl})$ and Normalized Confidence Interval Size (NCIS): The methodology involves the determination of the $\mathrm{Cl}$ for the risk curve obtained in the previous step using the delta method, an asymptotic normal approximation for the distribution of the estimated risk (Parr, 1983). The NCIS quantifies the mean and upper and lower $\mathrm{Cl}$ curves at discrete probabilities. It is defined as the ratio of the width of the $\mathrm{Cl}$ to the magnitude of the predictor variable/stimulus from the chosen model at a specific risk level. It is given by the following equation ( $U L$ and $L L$ represent the upper and lower limits of the $\mathrm{Cl}$ and $\mu$ represents the mean value of the metric at the chosen risk level). A lower NCIS magnitude is associated with a tighter confidence interval at the chosen probability level.

$$
N C I S_{\text {risk } \%}=\frac{\left(C I_{U L}-C I_{L L}\right)_{\text {risk } \%}}{\mu_{\text {risk } \%}}
$$

\section{Application to side-impact PMHS experiments}

The improved methodology is demonstrated using experiments conducted at the Medical College of Wisconsin, a dataset used to derive injury criteria for the ES2-re anthropomorphic 
Paper to be submitted to Journal of Biomechanics

212 test device (Kuppa et al., 2003). Briefly, experiments were conducted using whole body Post

213 Mortem Human Subjects (termed as specimens or PMHS) to determine side-impact responses

214 simulating automotive environments. Different velocities, padding and rigid load wall

215 conditions, offsets, and supplemental restraint systems, i.e., with and without side-impact

216 airbags, were used. Specimens were seated erect on a custom Teflon-coated seat, fixed to the

217 sled platform. A stationary load-wall was rigidly attached to the sled platform at the left end of

218 the seat, as viewed from its back. The sled delivered the pre-chosen impact velocity to the seat

219 and specimen. The specimen traversed laterally from right to left and contacted the load-wall

220 upon the application of the lateral acceleration pulse from the sled equipment. The test setup

221 is shown (Figure 2). Biological outcomes consisted of injury and non-injury information,

222 identified during autopsy and imaging techniques.

223

All specimens were subjected to single lateral impact loading. A total of 24 metrics were

225 chosen from 30 tests to demonstrate the feasibility of the proposed methodology (Table 2).

226 They included data from different types of sensors: accelerometers for the Thoracic Trauma

227 Index (TTI), peak pelvic acceleration and average spinal acceleration (ASA), and load cells for the

228 thoracic and pelvic forces. Non-injury outcomes were considered as right and injury outcomes

229 (based on MAIS 3+ classification) were considered as left censored data points. The left

230 censoring status was assigned because the timing of injuries to the thorax and abdomen could

231 not be precisely determined from experimental data.

232 


\section{Results}

Statistical analysis was conducted using the R-studio software, version 0.99 (R Studio, Inc.). The AROC ordered from highest to lowest is shown (Figure 3). Based on the paradigm shown in Figure 1, TTI was chosen as the most appropriate metric as it had the highest AROC that satisfied the KS p-value criterion. Table 3 shows the results of the analysis. The AIC outputs for the TTI metric were 36.57, 36.89, and 36.95 for the Weibull, log-normal and log-logistic distributions, and hence, the Weibull distribution represented the best distribution among those assessed. The influential observation was found to be associated with data points 22 for both shape and scale, and 25 for scale. However, none were deleted because they were deemed to be accurate from a biomechanical viewpoint. In other words, all data points or test numbers were considered in the subsequent survival analysis. The $95 \% \mathrm{Cl}$ curves obtained using the delta method and the mean risk curve for the predictor variable are shown (Figure 4). The NCIS at 5, 10, 20, 50, 80, and 90\% risks are given in Table 4.

\section{Discussion}

As stated in the introduction, the objective of this study was to develop an improved methodology to derive injury risk curves using survival analysis. An experimental dataset widely used for injury criteria and computational modeling was selected to demonstrate its feasibility (Kuppa et al., 2003; Vavalle et al., 2013). The methodology was At the outset it should be acknowledged that the ISO working group was tasked to recommend a process for determining injury criteria in the form of probability curves for automotive applications, and the consensus-driven approach was a result of a multi-year collaborative work from an international team of researchers (including the first author of this paper). The 
Paper to be submitted to Journal of Biomechanics

255

256

257

258

259

260

261

262

263

264

265

266

267

268

269

270

271

272

273

274

275 The input data selection involves identifications of likely injury metrics and status, stemming 276 from the same hypothesis, loading paradigm and injury mechanism and severity. It should be

recommendation of the working group (WG6 referred in the introduction section) has been used to derive risk curves from side-impact sled tests and foot-ankle-leg PMHS experiments (Petitjean et al., 2012; Yoganandan et al., 2015,a-b). While applying to other body regions and loading environments, it was deemed necessary to re-examine this approach and ensure that the methodology is clear, more general and robust. This was the impetus for the current study.

The use of survival analysis techniques is in accordance with the original recommendation because of the inclusion of censored data. Many metrics are routinely gathered and or derived during post processing from PMHS experiments, and the efficacy of the metric describing a complex biomechanical PMHS impact test is generally not known. The first step is therefore to choose the most appropriate metric from this pool that predicts injury outcomes. The identification of the most optimal metric based on the AROC output is an important and first deviation from the ISO approach. The present statistical analysis assists in the post processing phase, and may also have implications in designing future experiments of similar complexity and or loading. More importantly, however, it underscores the need to gather similar data from physical models if the overall experiment is designed to derive injury assessment risk curves for anthropomorphic test devices, routinely used to improve human safety and crashworthiness in automotive and military environments. The present methodology can therefore, be effectively used for dummy design, evaluation, injury criteria and human safety.

$$
\text { from the same hypothesis, loading paradigm and injury mechanism and severity. It should be }
$$


noted that all metrics should be available for each test from the entire experimental dataset (in this study a reduced set was used to ensure this feature). The selection of the group of metrics should be decided by the user. In the event of a missing data point for a specific metric, e.g., TTI, then (1) all data is removed for the subject/cadaver that has the missing TTI information or, (2) all TTI data is removed completely for all subjects/cadavers. The sample size decreases by one in the former option. In the latter option, the number of candidate metrics reduces while maintaining the sample size. This underscores the importance of gathering the full set of biomechanical metrics for each specimen, a factor not emphasized in the current ISO approach.

The assignment of censoring statuses for injuries and non-injuries are based on sensors used in biomechanical experiments: exact censoring used when injury timing is known from experiments, else, left censoring, and interval censoring for repeated tests on the same specimen. Exact censoring can be used with confidence if instruments such as strain gages and or acoustic emission sensors are included in the experimental design and the injury is well characterized using these sensors. For example, a strain gage sensor may provide surface profiles of local deformations of bone, it may not be fully adaptable if fractures initiate from distances farther away from its physical location and to soft tissue injuries such as ligaments and discs. The biomechanical community has yet to develop a consensus for processing acoustic emission signals, which depend on factors such as rate of load application and type of experimental model (isolated long bone, vertebral body, segmented spinal column), as widely varying methods have been suggested based on other disciplines and applied in biomechanical studies (Arun et al., 2014; Cormier et al., 2008; Cormier et al., 2011; Funk et al., 2002; Lockner, 
1993; McKay and Bir, 2009; Shridharani JK et al., 2014; Van Toen et al., 2012). From this perspective, it will be prudent to resort to the left censoring scheme to identify injury data points for the development of human injury probability curves from survival analysis. However, the left censored assignment for injury data results in lower estimates. This is a desirable feature for crashworthiness and safety applications.

The newly introduced procedure using the AROC and KS statistics provide important additional statistical information. For example, the AROC statistic is a single measure of the overall predictive accuracy of the model. It is free of distributional or model assumptions, while other parameters such as the Wald statistic used in biomechanics literatures relies on asymptotic approximations (Kuppa et al., 2000). With the same underlying sample, it is meaningful to compare the AROC between methods and different models, for evaluating predictive ability. The ROC curve is obtained by plotting the true positive rate against the false positive rate. The area of the perfect ROC curve is one. The ROC curve with an area of 0.5 indicates that the model is no better at predicting than random guesses.

A distinct advantage of the use of the AROC statistic is that in large datasets it could be made more robust by dividing the sample into training and test sets: the training set on which the survival analysis is fitted and the test set on which AROC is evaluated based on the fit, but was blinded (un-used) during the actual fitting. Cross validation analysis can be done using the heldout sample method which can be used to further reduce over-fitting the distribution (Picard and Cook, 1984; Shao, 1993). The AROC cannot be computed in certain cases, termed as edge 
Paper to be submitted to Journal of Biomechanics

cases. These edge cases involve experimental designs wherein all specimens are tested to injury levels, i.e., no non-injury data points. However, if such a situation arises, the adopted experimental design underscores the need to obtain additional data or alter the methodology such that a reasonable combination of injury and no injury data are obtained. If data acquisition precisely points to the time of injury and consist of only injury tests, the AROC parameter can be ignored for risk curve development for the specific metric associated with the timing issue. Because PMHS tests are often designed to extract more than one mechanical metric as shown in the demonstration dataset, other metrics may be considered left censored which should allow the use of AROC in a carefully planned experimental design.

The KS measures provide quantitative statistical information (Daniel, 1990). Hence, it was selected to assess the best underlying distribution for the specific biomechanical injury metric. The distance- based $p$-value reflects the difference between the fitted distribution and the nonparametric fit, a quantitative measure for the visual overlay plot, currently recommended in the ISO approach. These visual plots can be used in the qualitative diagnosis of a model. So, this feature is also maintained in the present method. The chosen $p$-value of 0.05 for the acceptance criterion for KS is often used, but, the end user can tailor the p-value to individual requirements.

The specification of the approximation method to determine the $\mathrm{Cl}$ of the injury risk curve further provides clarity and statistical robustness to the description of injury risk curves. The recommended delta method has better asymptotic accuracy and variance estimates than a 
343 direct normal approximation for the $\mathrm{Cl}$ (Parr, 1983). This process tends to produce wider 344 intervals at the tails, resulting in wider NCIS magnitudes. The risk curve confidence is therefore 345 more conservative than for a conventional logistic regression. This method has been applied in 346 recent studies as an extension of the ISO recommendation (Yoganandan et al., 2015; 347 Yoganandan et al., 2014).

349 The definition of the quality index for the $\mathrm{Cl}$ data was arbitrary in the ISO approach. In contrast, 350 the NCIS in the present methodology provides a quantitative measure so that the end user can 351 select different threshold values at discrete probabilities, or combine the NCIS data at different 352 risk levels or ranges to achieve individual goals. This feature is attractive in military 353 data/datasets. Further, no specific quality indices or their range are proposed because user(s) may have differing objectives. While the choice of the $95 \%$ interval is standard in many disciplines, the end user can alter this value. For example, the United States military can use 356 this method as its application is different from automotive crashworthiness. Thus, the 357 improved methodology is clearer, and has increased generalizability, flexibility and robustness 358 to derive most optimal human (PMHS) or physical model (dummy/manikin) injury probability 359 curves from a pool of fundamental/recorded and derived data/observations biomechanical 360 experiments. This process may be efficaciously used in retrospective and prospective analysis 361 of impact data from biomechanical tests and in future experimental designs for applications in different crashworthiness disciplines to advance human safety in different environments. 
Paper to be submitted to Journal of Biomechanics

364

365

366

367

368

369

370

371

372

373

374 this article are those of the authors and not necessarily representative of the funding 375 organizations.

376

377 Role of study sponsors: No authors are sponsors of the study. 
Paper to be submitted to Journal of Biomechanics

$378 \quad$ Figure captions

379

380 Figure 1: Flow chart of the statistical process to derive risk curves.

381

382 Figure 2: Schematic of the sled test set up for PMHS experiments.

383

384

Figure 3: AROC rank ordered by metric.

385

386 Figure 4a: A. Injury risk curve for selected metric, TTI, showing 95\% confidence intervals.

387

388 Figure 4b: Dfbeta plots

389

390

Figure 4c: Q-Q plot of the resulting selection.

391 
Table 1: Data from PMHS sled tests (Kuppa et al., 2003)

\begin{tabular}{|c|c|c|c|c|c|c|c|c|c|c|c|c|c|c|c|c|c|c|c|c|c|c|c|c|c|}
\hline ID & thx_f & $a b d \_f$ & pel_f & Hdmax & Hdavg & $H V \max$ & HVC & Fdmax & Fdavg & $F V \max$ & $\mathrm{FVC}$ & HVavg & HVCavg & FVavg & FVCavg & TTI & ASA & spu & $\mathrm{spl}$ & rspu & $\mathrm{rspl}$ & rlu & rll & pel & MAIS* \\
\hline 6 & 13033 & 3139 & 6893 & 0.176 & 0.173 & 8.6 & 1.1 & 0.299 & 0.267 & 16.8 & 1.1 & 6.13 & 0.81 & 14.78 & 0.95 & 154 & 45 & 51 & 54 & 54 & 56 & 135 & 174 & 57 & 2 \\
\hline 15 & 7487 & 3525 & 8663 & 0.271 & 0.226 & 5.3 & 1 & 0.412 & 0.332 & 8.8 & 2.1 & 4.49 & 0.74 & 7.39 & 1.72 & 170 & 63 & 73 & 93 & 74 & 112 & 60 & 130 & 51 & 3 \\
\hline 31 & 8682 & 3759 & 7745 & 0.281 & 0.262 & 6.1 & 1.1 & 0.406 & 0.366 & 6.7 & 2.7 & 5.64 & 0.96 & 6.47 & 2.04 & 178 & 54 & 56 & 91 & 61 & 95 & 89 & 161 & 56 & 4 \\
\hline 32 & 9025 & 3584 & 8556 & 0.274 & 0.224 & 4.6 & 0.9 & 0.421 & 0.343 & 6.2 & 2.8 & 4.26 & 0.75 & 5.6 & 2.08 & 180 & 56 & 71 & 62 & 77 & 64 & 129 & 194 & 51 & 4 \\
\hline 33 & 6504 & 4303 & 9153 & 0.268 & 0.213 & 11.5 & 2 & 0.411 & 0.365 & 16.1 & 6.9 & 9.75 & 1.63 & 13.3 & 4.97 & 153 & 51 & 58 & 57 & 77 & 64 & 114 & 46 & 44 & 3 \\
\hline 4 & 3498 & 1970 & 5618 & 0.114 & 0.104 & 3.3 & 0.3 & 0.176 & 0.163 & 5.2 & 0.5 & 2.87 & 0.24 & 4.78 & 0.46 & 154 & 72 & 23 & 68 & 24 & 70 & 37 & 62 & 32 & 0 \\
\hline 14 & 4160 & 2301 & 5190 & 0.32 & 0.264 & 5.3 & 1.2 & 0.409 & 0.356 & 6.7 & 1.5 & 4.56 & 0.96 & 6.21 & 1.23 & 142 & 34 & 46 & 29 & 49 & 34 & 63 & 111 & 37 & 4 \\
\hline 18 & 3691 & 2187 & 7869 & 0.214 & 0.185 & 4.7 & 0.8 & 0.318 & 0.259 & 6.6 & 1.3 & 3.77 & 0.64 & 4.89 & 0.87 & 153 & 30 & 42 & 51 & 48 & 53 & 81 & 106 & 35 & 3 \\
\hline 21 & 4133 & 2018 & 7893 & 0.203 & 0.148 & 4 & 0.6 & 0.305 & 0.253 & 6.2 & 1.2 & 3.16 & 0.36 & 5.05 & 0.87 & 155 & 23 & 33 & 38 & 34 & 39 & 92 & 111 & 35 & 1 \\
\hline 35 & 3046 & 1634 & 6012 & 0.275 & 0.235 & 4.2 & 0.8 & 0.384 & 0.320 & 4.8 & 1.5 & 3.61 & 0.57 & 3.99 & 1.08 & 133 & 16 & 22 & 37 & 22 & 38 & 92 & 166 & 51 & 2 \\
\hline 16 & 3482 & 758 & 9067 & 0.107 & 0.075 & 2.5 & 0.1 & 0.191 & 0.148 & 4.1 & 0.4 & 2.48 & 0.14 & 3.4 & 0.3 & 129 & 28 & 26 & 31 & 27 & 32 & 44 & 65 & 23 & 2 \\
\hline 7 & 16338 & 3888 & 9905 & 0.239 & 0.219 & 7.1 & 1.9 & 0.395 & 0.364 & 10.9 & 2.2 & 6.68 & 1.44 & 10.16 & 1.83 & 158 & 51 & 94 & 62 & 94 & 90 & 88 & 140 & 76 & 2 \\
\hline 8 & 7225 & 3668 & 19104 & 0.292 & 0.225 & 10.9 & 2.3 & 0.41 & 0.346 & 9.7 & 2.1 & 8.57 & 1.46 & 9.2 & 1.85 & 172 & 34 & 111 & 74 & 117 & 87 & 135 & 125 & 60 & 4 \\
\hline 11 & 4395 & 2393 & 21071 & 0.27 & 0.270 & 3.9 & 1 & 0.37 & 0.370 & 7.2 & 1.2 & 3.16 & 0.42 & 6.33 & 0.75 & 202 & 71 & 56 & 54 & 57 & 55 & 132 & 106 & 66 & 3 \\
\hline 12 & 7447 & 2250 & 19336 & 0.291 & 0.255 & 6.8 & 1.5 & 0.412 & 0.376 & 11.8 & 2.6 & 6.44 & 1.29 & 10.93 & 2.2 & 231 & 125 & 103 & 103 & 112 & 109 & 142 & 164 & 67 & 5 \\
\hline 3 & 4975 & 5314 & 3372 & 0.243 & 0.212 & 6 & 1.4 & 0.394 & 0.350 & 7.3 & 1.9 & 5.51 & 1.01 & 6.74 & 1.52 & 160 & 26 & 49 & 51 & 50 & 57 & 183 & 112 & 55 & 3 \\
\hline 19 & 4997 & 3095 & 5945 & 0.215 & 0.189 & 5.5 & 0.8 & 0.374 & 0.317 & 9.2 & 2.2 & 4.97 & 0.64 & 8.33 & 1.8 & 164 & 46 & 52 & 63 & 54 & 66 & 102 & 95 & 41 & 0 \\
\hline 20 & 5744 & 3414 & 8972 & 0.235 & 0.215 & 5.2 & 0.8 & 0.364 & 0.325 & 6.7 & 1.7 & 4.58 & 0.6 & 6.47 & 1.25 & 195 & 33 & 47 & 41 & 47 & 50 & 183 & 67 & 46 & 3 \\
\hline 29 & 4773 & 2725 & 9350 & 0.217 & 0.152 & 6.7 & 1 & 0.329 & 0.221 & 6.4 & 1.6 & 4.96 & 0.6 & 5.22 & 0.97 & 121 & 17 & 82 & 45 & 82 & 53 & 156 & 167 & 58 & 0 \\
\hline 30 & 2992 & 2735 & 5456 & 0.293 & 0.256 & 17.6 & 3.9 & 0.431 & 0.391 & 26 & 11.8 & 13.94 & 3 & 22.91 & 9.5 & 167 & 22 & 77 & 66 & 77 & 89 & 163 & 278 & 47 & 4 \\
\hline 34 & 3769 & 1990 & 7339 & 0.266 & 0.239 & 6 & 1.2 & 0.407 & 0.332 & 6.4 & 1.7 & 5.21 & 0.98 & 5.57 & 1.32 & 142 & 57 & 48 & 59 & 49 & 60 & 121 & 102 & 46 & 4 \\
\hline 36 & 3896 & 2648 & 10891 & 0.207 & 0.177 & 11.9 & 2 & 0.332 & 0.278 & 15.5 & 3.2 & 10.45 & 1.55 & 14.07 & 2.7 & 181 & 81 & 66 & 80 & 72 & 84 & 114 & 111 & 57 & 2 \\
\hline 22 & 4256 & 8083 & 4744 & 0.074 & 0.066 & 3.4 & 0.2 & 0.117 & 0.103 & 4.4 & 0.4 & 2.8 & 0.14 & 3.79 & 0.3 & 150 & 41 & 39 & 41 & 44 & 47 & 121 & 115 & 42 & 3 \\
\hline 24 & 3891 & 7236 & 3413 & 0.144 & 0.135 & 4.7 & 0.5 & 0.2 & 0.186 & 5.3 & 0.9 & 4.37 & 0.41 & 4.96 & 0.71 & 143 & 43 & 44 & 61 & 52 & 64 & 115 & 130 & 88 & 2 \\
\hline 26 & 1704 & 6905 & 2847 & 0.22 & 0.190 & 7.9 & 0.8 & 0.33 & 0.300 & 9.8 & 1.9 & 4.92 & 0.58 & 7.4 & 1.41 & 119 & 23 & 57 & 50 & 61 & 52 & 70 & 99 & 62 & 3 \\
\hline 9 & 3657 & 559 & 10838 & 0.279 & 0.245 & 5.5 & 0.9 & 0.359 & 0.324 & 8.3 & 1.4 & 4.77 & 0.72 & 7.15 & 1.1 & 189 & 33 & 79 & 35 & 80 & 38 & 139 & 44 & 54 & 4 \\
\hline 28 & 9329 & 532 & 7787 & 0.184 & 0.158 & 9 & 1 & 0.25 & 0.220 & 12.2 & 1.9 & 7.93 & 0.87 & 10.56 & 1.57 & 124 & 11 & 56 & 41 & 63 & 49 & 90 & 94 & 41 & 2 \\
\hline 38 & 5112 & 1680 & 9229 & 0.154 & 0.120 & 3 & 0.2 & 0.229 & 0.200 & 3.6 & 0.5 & 2.52 & 0.14 & 2.75 & 0.38 & 111 & 16 & 22 & 18 & 27 & 46 & 28 & 46 & 53 & 0 \\
\hline 41 & 4280 & 1930 & 9998 & 0.284 & 0.230 & 6.5 & 1.2 & 0.384 & 0.320 & 9.5 & 2 & 6.06 & 0.93 & 8.5 & 1.62 & 116 & 23 & 22 & 36 & 26 & 37 & 29 & 43 & 60 & 2 \\
\hline 42 & 3078 & 2358 & 7226 & 0.16 & 0.150 & 8 & 0.9 & 0.272 & 0.230 & 12.2 & 3 & 7.06 & 0.8 & 10.44 & 2.06 & 146 & 37 & 28 & 47 & 33 & 50 & 26 & 40 & 51 & 2 \\
\hline
\end{tabular}


Table 2. Metric definitions from Table 1 (Kuppa et al., 2003)

\begin{tabular}{|c|c|c|c|c|c|}
\hline Metric & Unit & Definition & Metric & Unit & Definition \\
\hline abd_f & $\mathrm{N}$ & Normalized max abdominal wall force & HVCavg & $\mathrm{m} / \mathrm{s}$ & Same as FVCavg but with half-width \\
\hline ASA & g & Average spinal acceleration & Hvmax & $\mathrm{m} / \mathrm{s}$ & Same as Fvmax but with half-width \\
\hline Fdavg & $\mathrm{mm}$ & Average full-width deflection, normalized & pel & g & Pelvis acceleration \\
\hline Fdmax & $\mathrm{mm}$ & Max full-width deflection, normalized & pel_f & $\mathrm{N}$ & Normalized max pelvis wall force \\
\hline Fvavg & $\mathrm{m} / \mathrm{s}$ & Average velocity of full-width deflection measure & rll & g & Normalized left lower rib acceleration \\
\hline FVC & $\mathrm{m} / \mathrm{s}$ & Max velocity*compression derived from full width & rlu & g & Normalized left upper rib acceleration \\
\hline FVCavg & $\mathrm{m} / \mathrm{s}$ & Average velocity*compression derived from full width & rspl & g & Normalized resultant lower spine acceleration \\
\hline Fvmax & $\mathrm{m} / \mathrm{s}$ & Max velocity from full-width deflection measure & rspu & g & Normalized resultant upper spine acceleration \\
\hline Hdavg & Ratio & Same as Fdavg but with half-width & spl & g & Normalized lateral lower spine acceleration \\
\hline Hdmax & Ratio & Same as Fdmax but with half-width & spu & g & Normalized lateral upper spine acceleration \\
\hline Hvavg & $\mathrm{m} / \mathrm{s}$ & Same as Fvavg but with half-width & the_f & $\mathrm{N}$ & Normalized maximum thoracic wall force \\
\hline HVC & $\mathrm{m} / \mathrm{s}$ & Same as FVC but with half-width & TTI & g & Thoracic trauma index \\
\hline
\end{tabular}


Table 3: Summary of statistical results

\begin{tabular}{|c|c|c|c|c|c|c|c|c|}
\hline \multirow{2}{*}{ No } & \multirow{2}{*}{ Variable } & \multirow{2}{*}{ AROC } & \multicolumn{3}{|c|}{ AIC } & \multicolumn{3}{|c|}{ KS p value } \\
\hline & & & LL & LN & WB & LL & LN & WB \\
\hline 3 & Fdavg & 0.857 & 39.19 & 39.92 & 37.62 & $<0.05$ & $<0.05$ & $<0.05$ \\
\hline 9 & Hdavg & 0.839 & 38.33 & 39.12 & 36.62 & $<0.05$ & $<0.05$ & $<0.05$ \\
\hline 4 & Fdmax & 0.830 & 40.59 & 41.11 & 39.26 & $<0.05$ & $<0.05$ & $<0.05$ \\
\hline 10 & Hdmax & 0.826 & 39.32 & 40.03 & 37.81 & $<0.05$ & $<0.05$ & $<0.05$ \\
\hline 24 & TTI & 0.763 & 36.95 & 36.89 & 36.57 & Not computed & Not computed & 0.2365 \\
\hline
\end{tabular}

Results after applying statistical paradigm presented in Figure 1. The selected metric, TTI, is shown in bold. P-values below $1 \times 10^{-4}$ are shown as zero. Results are shown for metrics in the order of the highest AROC and following the flow chart shown (Figure 1), results the analysis is stopped after meeting all specified criteria. WB was used for the TTI metric to derive the risk curve and obtain NCIS because the AIC was the lowest. Hence, it was not necessary to compute the KS statistic for the LL and LN. LL: log logistic, LN: log normal, WB: Weibull, KS - KolmogorovSmirnov 
Paper to be submitted to Journal of Biomechanics

Table 4: Normalized confidence interval size (NCIS) and data for the TTI (g) metric based on Weibull distribution, survival analysis.

\begin{tabular}{|c|c|c|c|c|}
\hline Risk Level & NCIS & $\begin{array}{c}\text { Lower } \\
\text { Bound }\end{array}$ & Mean & $\begin{array}{c}\text { Upper } \\
\text { Bound }\end{array}$ \\
\hline 0.05 & 0.87 & 62 & 95 & 145 \\
\hline 0.25 & 0.38 & 108 & 130 & 158 \\
\hline 0.50 & 0.21 & 137 & 153 & 170 \\
\hline 0.90 & 0.34 & 161 & 190 & 225 \\
\hline 0.95 & 0.40 & 163 & 199 & 243 \\
\hline
\end{tabular}




\section{References}

Arbogast KB, Maltese MR. 2015. Pediatric injury biomechanics. In: N Y, A.M. N, Melvin JW, editors. Accidental Injury: Biomechanics and Prevention, Third ed. NY: Springer,. p 643-696.

Arun MW, Yoganandan N, Stemper BD, Pintar FA. 2014. A methodology to condition distorted acoustic emission signals to identify fracture timing from human cadaver spine impact tests. J Mech Behav Biomed Mater 40:156-160.

Belsley DA, Kuh E, Welsch RE. 1980. Regression diagnostics: Identifying influential data and sources of collinearity Wiley, New York.

Cavanaugh JH, Yoganandan N. 2015. Thoracic injury biomechanics. In: N Y, A.M. N, Melvin JW, editors. Accidental Injury: Biomechanics and Prevention, Third ed. NY: Springer,. p 331-372.

Cormier J, Manoogian S, Bisplinghoff J, McNally C, Duma S. 2008. The use of acoustic emission in facial fracture detection. Biomed Sci Instrum 44:147-152.

Cormier J, Manoogian S, Bisplinghoff J, Rowson S, Santago A, McNally C, Duma S, Bolte Jt. 2011. The tolerance of the maxilla to blunt impact. J Biomech Eng 133:064501.

Cutcliffe HC, Schmidt AL, Lucas JE, Bass CR. 2012. How few? Bayesian statistics in injury biomechanics. Stapp Car Crash J $56: 349-386$.

Daniel WW. 1990. Daniel, Wayne W. (1990). "Kolmogorov-Smirnov one-sample test". Applied Nonparametric Statistics., Second ed. Boston, MA.: PWS-Kent.

Findley DF, Ching-Zong W. 2002. AIC, Overfitting Principles, and the Boundedness of Moments of Inverse Matrices for Vector Autotregressions and Related Models. Journal of Multivariate Analysis 83:415-450.

Funk JR, Crandall JR, Tourret LJ, MacMahon CB, Bass CR, Patrie JT, Khaewpong N, Eppinger RH. 2002. The axial injury tolerance of the human foot/ankle complex and the effect of Achilles tension. Journal of Biomechanical EngineeringTransactions of the Asme 124:750-757.

Guo G, Rodriguez G. 1992. Estimating a multivariate proportional hazards model for clustered data using the EM algorithm, with an application to child survival in Guatemala. Journal of the American Statistical Association 87:969-976.

Kent R, Funk J. 2004. Data Censoring and Parametric Distribution Assignment in the Development of Injury Risk Functions from Biomechanical Data," SAE Technical Paper 2004-01-0317, 2004, doi: 10.4271/2004-01-0317.

Kuppa S, Eppinger F, Maltese M, Naik R, Yoganandan N, Pintar F, Saul R, McFadden J. 2000. Assessment of thoracic injury criteria for side impact. In: Proceedings of the International Research Council on Biomechanics of Injury. Montpellier, France. p 131-146. 
Paper to be submitted to Journal of Biomechanics

Kuppa S, Eppinger RH, McKoy F, Nguyen T, Pintar FA, Yoganandan N. 2003. Development of Side Impact Thoracic Injury Criteria and Their Application to the Modified ES-2 Dummy with Rib Extensions (ES-2re). Stapp Car Crash J 47:189210.

Lockner D. 1993. The Role of Acoustic Emission in the Study of Rock Fracture. Int. J. Rock Mech. Min. Sci. \& Geomech. Abstr. 30:883-899.

McKay BJ, Bir CA. 2009. Lower extremity injury criteria for evaluating military vehicle occupant injury in underbelly blast events. Stapp Car Crash J 53:229-249.

Parr WC. 1983. A note on the jackknife, the bootstrap and the delta method estimators of bias and variance. Biometrika 70:719722.

Petitjean A, Torsseille X, N Y, Pintar FA. 2015. Normalization and scaling for human response corridors and development of risk curves In: N Y, A.M. N, Melvin JW, editors. Accidental Injury: Biomechanics and Prevention, Third ed. NY: Springer,. p 769-792.

Petitjean A, Trosseille X, Praxl N, Hynd D, Irwin A. 2012. Injury risk curves for the WorldSID 50th male dummy. Stapp Car Crash J 56:323-347.

Philippens M, Wismans J, Forbes PA, Yoganandan N, Pintar FA, Soltis SJ. 2009. ES2 neck injury assessment reference values for lateral loading in side facing seats. Stapp Car Crash J 53:421-441.

Picard R, Cook D. 1984. Cross-validation of regression models. Journal of the American Statistical Association 79:579-583.

Royston P, Parmar MK. 2002. Flexible parametric proportional-hazards and proportional-odds models for censored survival data, with application to prognostic modelling and estimation of treatment effects. Stat Med 21:2175-2197.

Rupp J. 2015. Knee, thigh and hip injury biomechanics. In: N Y, A.M. N, Melvin JW, editors. Accidental Injury: Biomechanics and Prevention, Third ed. NY: Springer,. p 471-497.

Salzar RS, LIevers BW, Bailey AM, Crandall JR. 2015. Leg, foot and ankle injury biomechanics. In: N Y, A.M. N, Melvin JW, editors. Accidental Injury: Biomechanics and Prevention, Third ed. NY: Springer,. p 499-548.

Seipel RC, Pintar FA, Yoganandan N, Boynton MD. 2001. Biomechanics of calcaneal fractures: a model for the motor vehicle. Clin Orthop Relat Res:218-224.

Shao J. 1993. Linear model selection by cross-validation. Journal of the American Statistical Association 88:486-494.

Shridharani JK, Schmidt AL, Cox CA, Bigler BR, Knight AE, CR B. 2014. Dynamic Failure Localization in Spinal Specimens using Acoustic Emissions, International Research Conference on the Biomechanics of Impact, September, Berlin.

Van Toen C, Street J, Oxland TR, Cripton PA. 2012. Acoustic emission signals can discriminate between compressive bone fractures and tensile ligament injuries in the spine during dynamic loading. J Biomech 45:1643-1649. 
Vavalle NA, Moreno DP, Rhyne AC, Stitzel JD, Gayzik FS. 2013. Lateral impact validation of a geometrically accurate full body finite element model for blunt injury prediction. Ann Biomed Eng 41:497-512.

Wang M, Rao R, Yoganandan N, Pintar FA. 2015. Upper extremity injury biomechanics. In: N Y, A.M. N, Melvin JW, editors. Accidental Injury: Biomechanics and Prevention, Third ed. NY: Springer,. p 309-330.

Yoganandan N, Arun MW, Pintar FA, Banerjee A. 2015. Lower Leg Injury Reference Values and Risk Curves from Survival Analysis for Male and Female Dummies: Meta-analysis of Postmortem Human Subject Tests. Traffic Inj Prev 16 Suppl $1:$ S100-107.

Yoganandan N, Pintar F, Banerjee A, Schlick M, Chirvi S, Uppal H, Merkle A, Voo L, Kleinberg M. Hybrid III Lower Leg Injury Assessment Reference Curves Under Axial Impacts Using Matched-Pair Tests. Biomed Sci Instrum 2015.51:230-237

Yoganandan N, Arun MW, Pintar FA, Szabo A. 2014. Optimized lower leg injury probability curves from postmortem human subject tests under axial impacts. Traffic Inj Prev 15 Suppl 1:S151-156.

Yoganandan N, Chirvi S, Pintar FA, Uppal H, Schlick M, Banerjee A, Voo L, Merkle A, Kleinberger M. 2016. Foot-Ankle Fractures and Injury Probability Curves from Post-mortem Human Surrogate Tests. Ann Biomed Eng.

Yoganandan N, Stemper BD, Pintar FA, Maiman DJ, McEntire BJ, Chancey VC. 2013. Cervical spine injury biomechanics: Applications for under body blast loadings in military environments. Clin Biomech (Bristol, Avon) 28:602-609. 
Figure 1

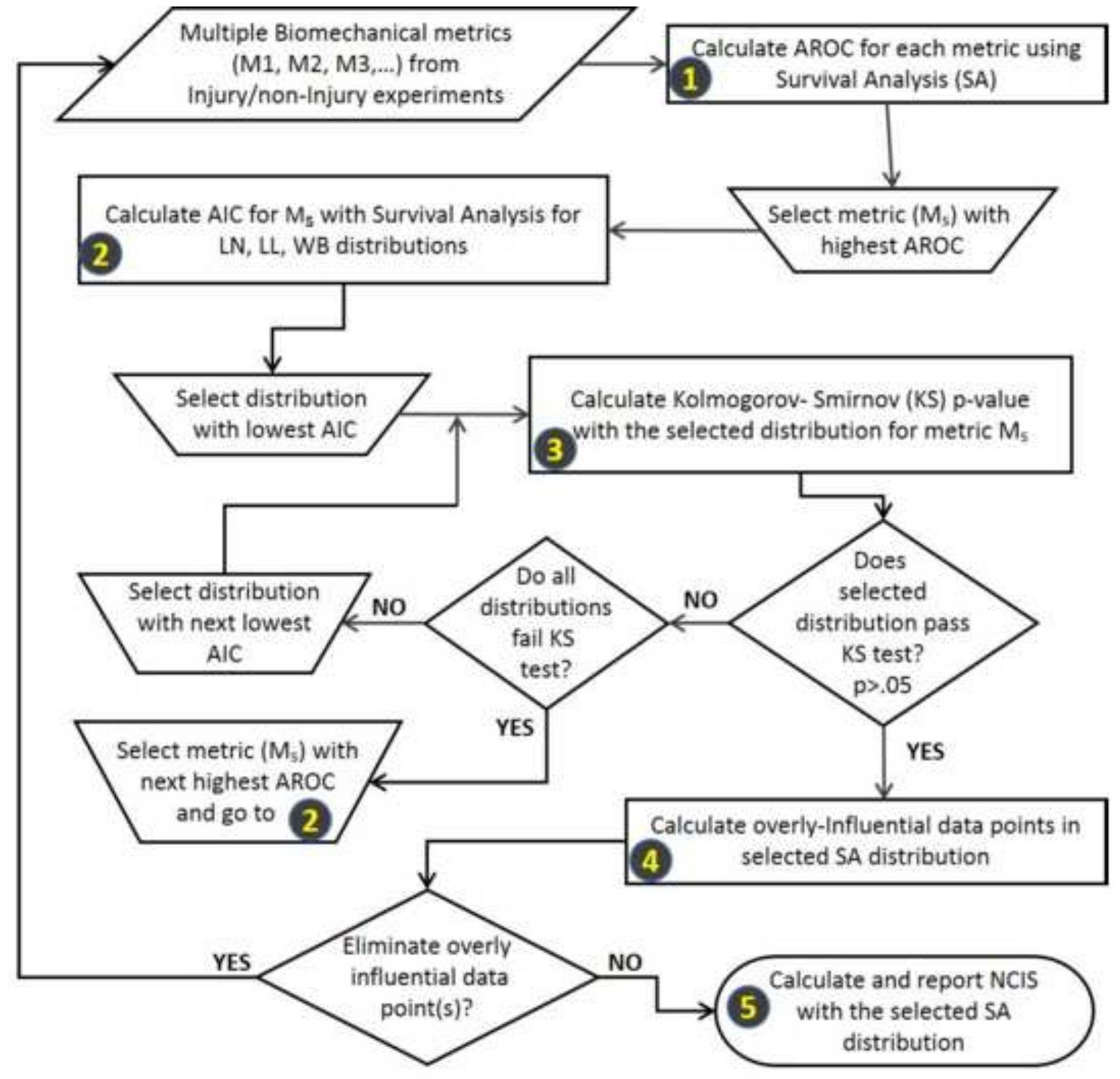

Figure 1: Flow chart of the statistical process to dertve risk curves. 


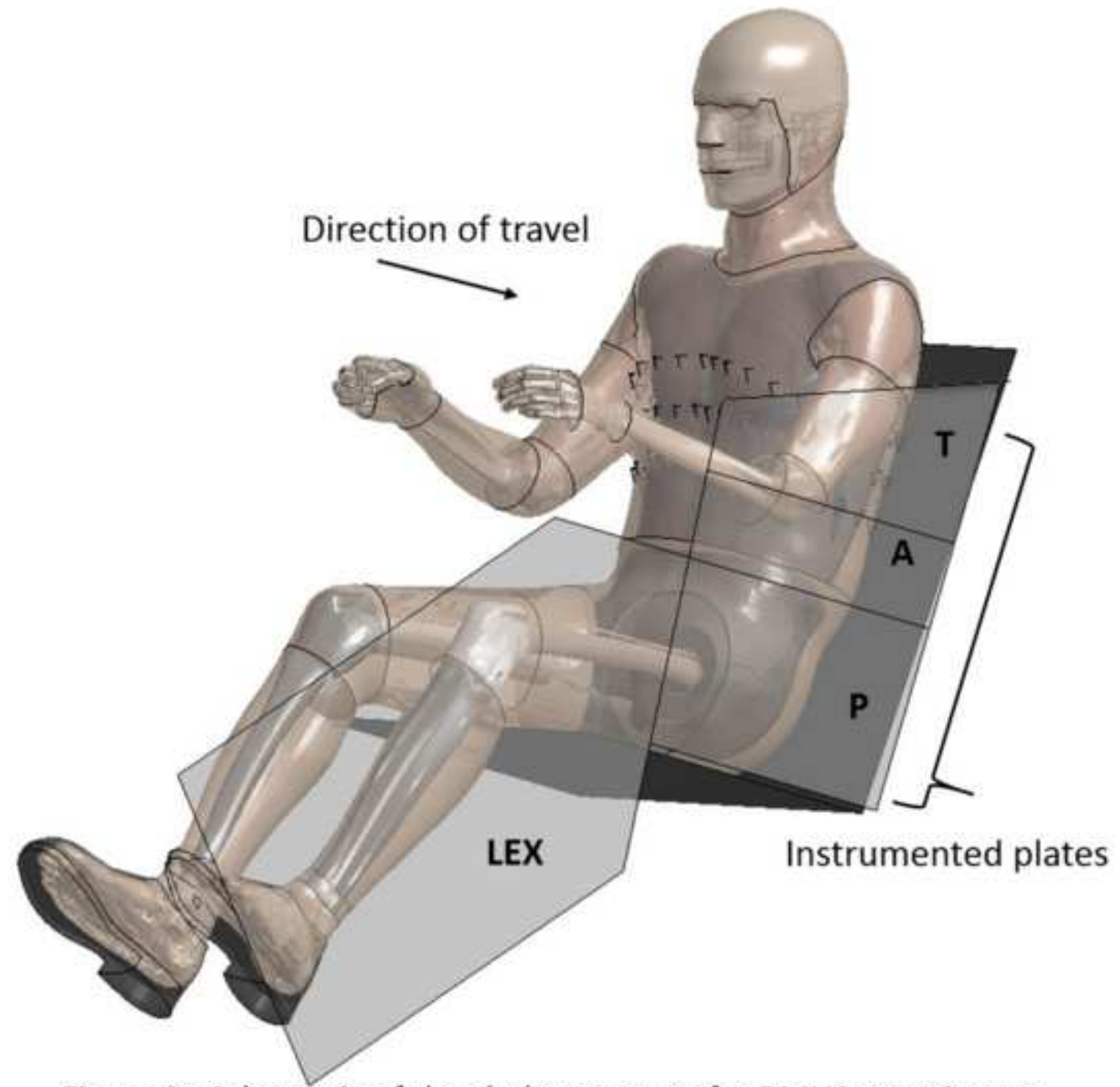

Figure 2: Schematic of the sied test set up for PMHS experiments.

Direction of travel

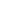




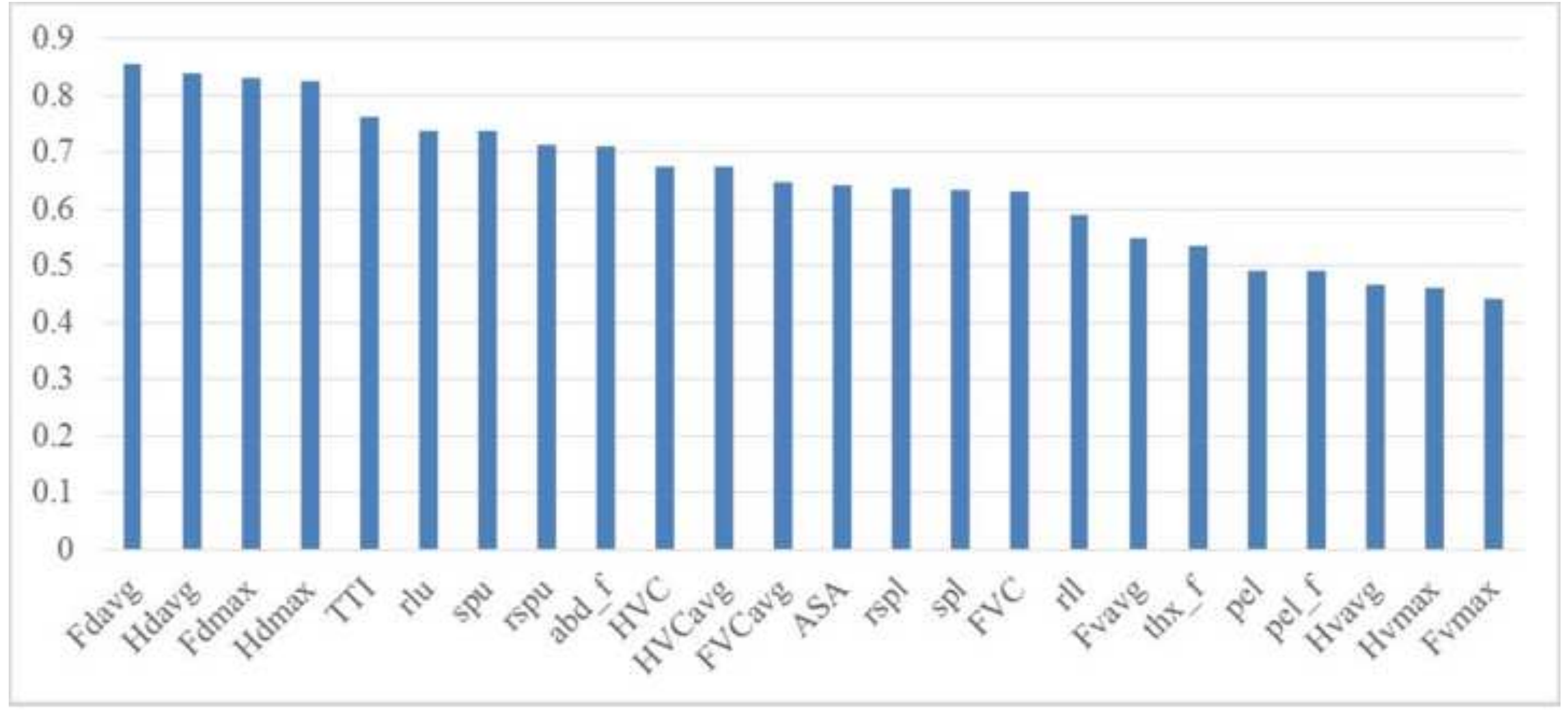

\section{Figure 3: AROC rank ordered by metric.}



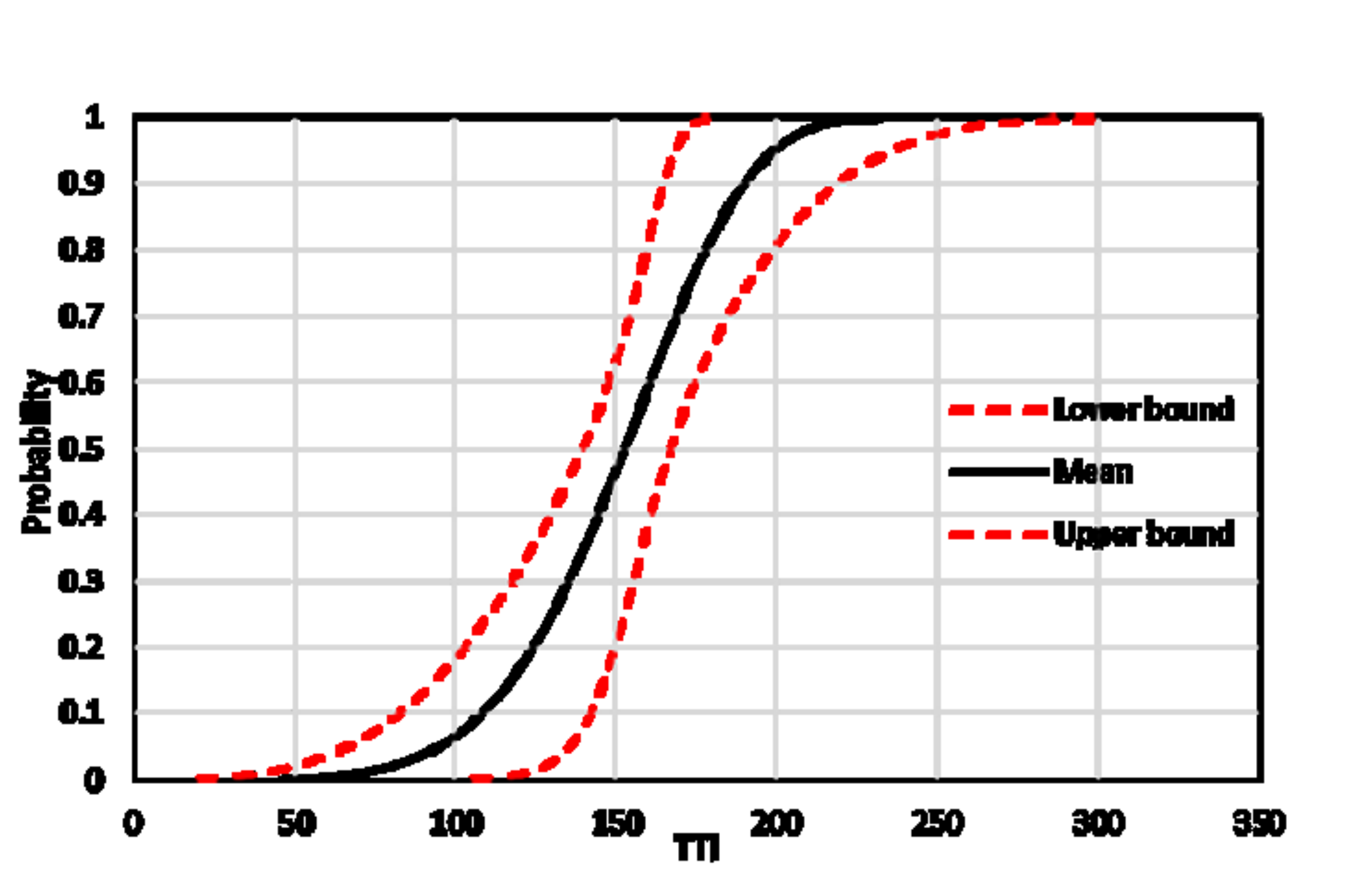

.
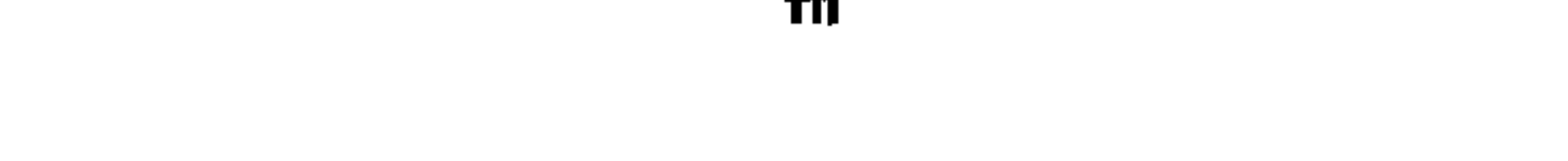


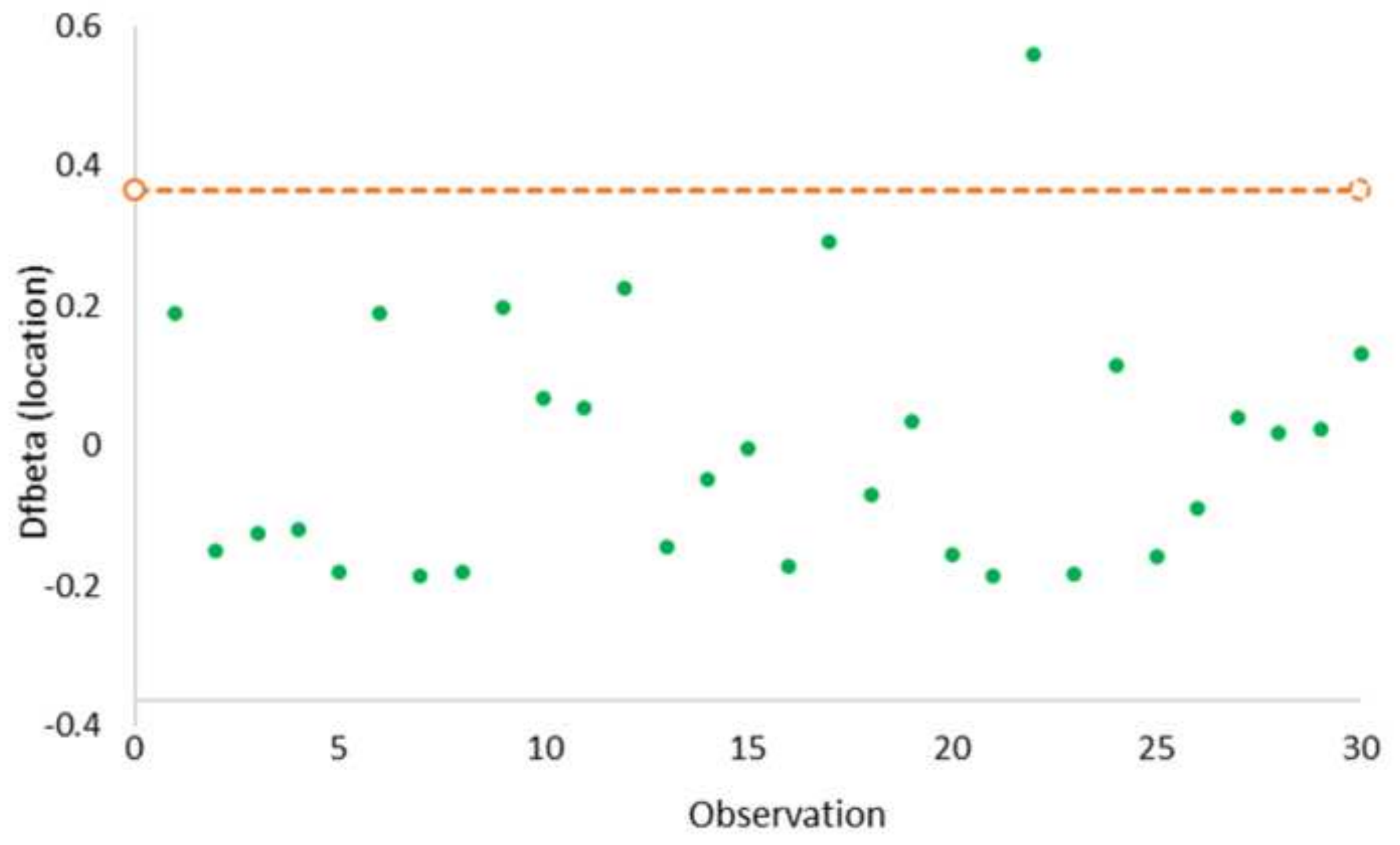




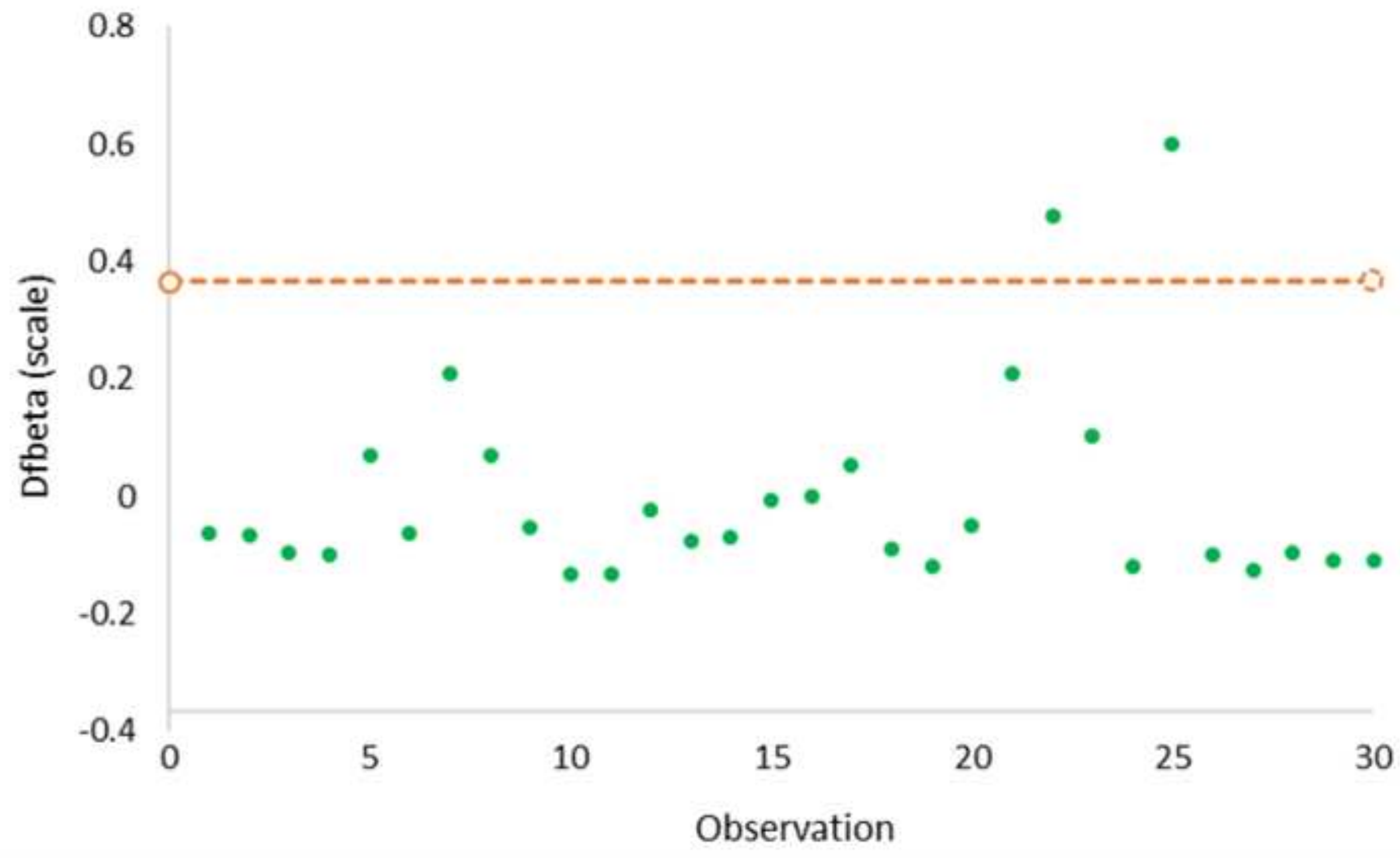




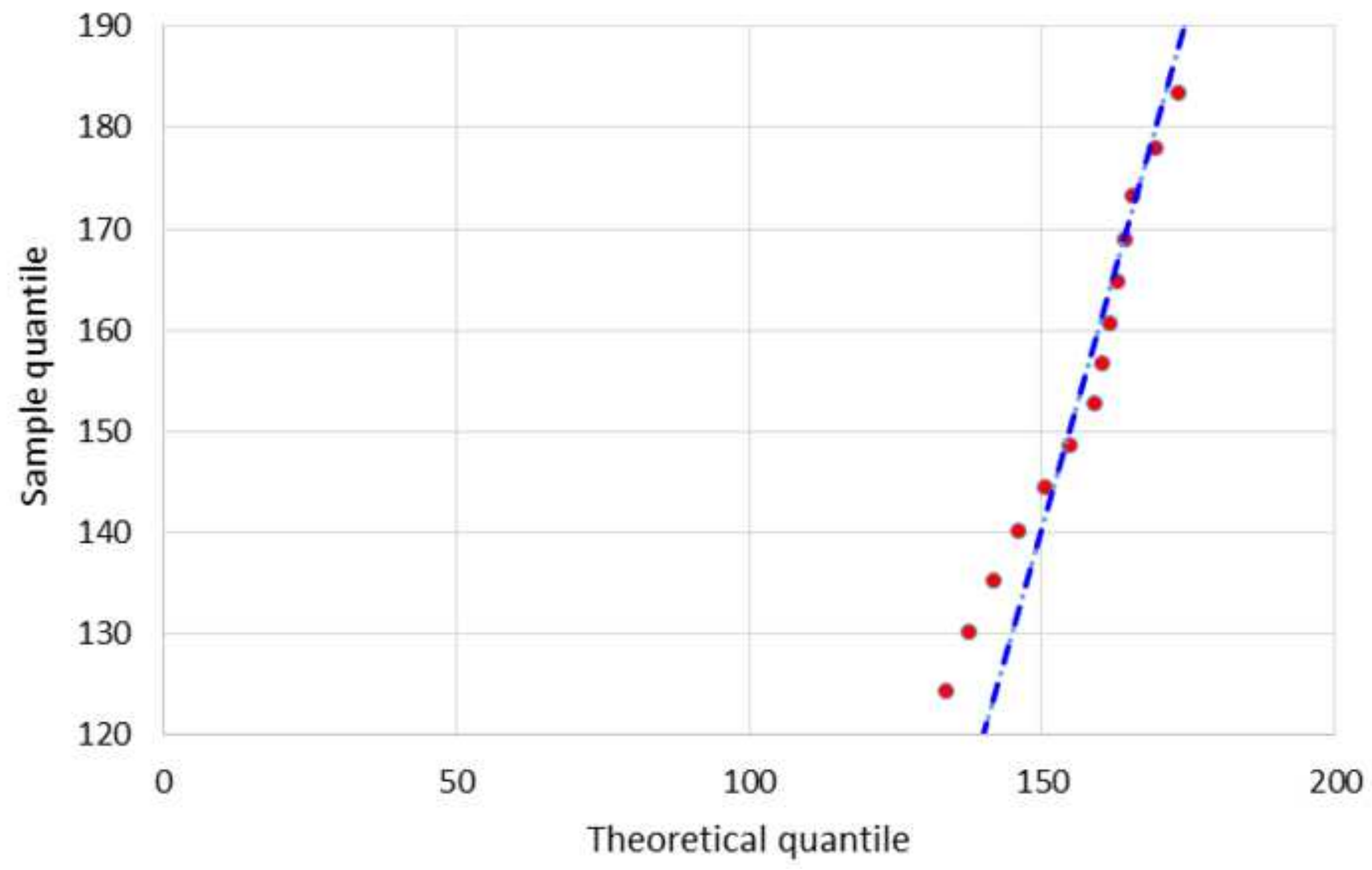

